\title{
Supersensitivity of muscarinic receptors in rat isolated detrusor smooth muscle (DSM) after chronic nitric oxide inhibition Fabiola Mónica*1, Gilberto de Nucci ${ }^{1}$, Alice Bricola ${ }^{2}$, Angelina Zanesco ${ }^{3}$, Catarina Porto ${ }^{4}$, Fernando Abdalla ${ }^{5}$, Leandro Freitas 6 , Maria Aparecida Teixeira ${ }^{7}$ and Edson Antunes ${ }^{1}$
}

\author{
Address: ${ }^{1}$ Department of Pharmacology, Faculty of Medical Sciences, University of Campinas, Campinas (SP), Brazil, ${ }^{2}$ Department of \\ Pharmacology, Pontifical Catholic University of Campinas, Campinas (SP), Brazil, ${ }^{3}$ Department of Physical Education; Institute of Bioscience, \\ Paulista State University, Rio Claro (SP), Brazil, ${ }^{4}$ Section of Experimental Endocrinology, Department of Pharmacology, UNIFESP (SP), Sao Paulo, \\ Brazil, ${ }^{5}$ Pharmacology Laboratory, Butanta Institute (SP), Sao Paulo, Brazil, ${ }^{6}$ Departament of Clinical Pathology, University of Campinas, \\ Campinas (SP), Brazil and ${ }^{7}$ Faculty of Medicine, Pontifical Catholic University of Campinas, Campinas (SP), Brazil \\ Email: Fabiola Mónica* - fabiolamonica@terra.com.br \\ * Corresponding author
}

from 3rd International Conference on cGMP Generators, Effectors and Therapeutic Implications Dresden, Germany. 15-17 June 2007

Published: 25 July 2007

BMC Pharmacology 2007, 7(Suppl I):P4 I doi:I0.|I86/|47|-22I0-7-SI-P4 I

This abstract is available from: http://www.biomedcentral.com/I47I-22 I0/7/SI/P4 I

(C) 2007 Mónica et al; licensee BioMed Central Ltd.

\section{Background}

It has been suggested that disturbance of the NO-cGMP pathway lead to impaired relaxation of the urethral outflow region, increased bladder afferent activity and overactive bladder, but the precise role of $\mathrm{NO}$ in regulating the detrusor smooth muscle (DSM) functions remains to be determined.

\section{Purpose}

Our present work aimed to examine the functional and biochemical alterations of rat DSM after chronic NO blockade.

\section{Methods}

Male Wistar rats were treated orally with L-NAME $(20 \mathrm{mg} /$ rat/day) for 30 days. Age-matched control animals received tap water alone. Concentration-response curves to full agonist carbachol (CCh, $1 \mathrm{nM}-30 \mu \mathrm{M})$ in the DSM were obtained. The values of potency $\left(\mathrm{pEC}_{50}\right)$ and maximal responses $\left(\mathrm{E}_{\max }\right)$ were calculated. The $\mathrm{IP}_{3}$ accumulation and the nitric oxide synthase (NOS) activity, as well as morphometric analyses were evaluated in the urinary bladder of control and L-NAME-treated rats.

\section{Results}

L-NAME-treated rats presented a marked arterial hypertension (ctrl:124 \pm 2 vs treated: $198 \pm 1 \mathrm{mmHg}$ ) and a reduction of $86 \%$ in the total NOS activity in the isolated rat bladder. Four-weeks treatment with L-NAME increased by 5 -fold the CCh potency $(6.09 \pm 0.02$ vs $6.82 \pm 0.06)$, without modifying the $\mathrm{E}_{\max }$ (ctl: $3.50 \pm 0.10$ vs treated: $3.40 \pm 0.07$ ) (Figure 1A). Incubation of urinary bladder with CCh $\left(10^{-9}-10^{-3} \mathrm{M}\right)$ concentration-dependently increased the total $\left[{ }^{3} \mathrm{H}\right]$-inositol phosphates in rat urinary bladder that was markedly higher in L-NAME-treated rats compared with control animals (Figure 1B). The measurement of the thickness of rat isolated bladder revealed that L-NAME treatment for 30 days caused no alterations in the thickness of submucosa and muscular layers of the DSM when compared with control animals. However, in the trigone smooth muscle (TSM), L-NAME treatment significantly increased the thickness of the muscular layer without changing the thickness of the sub-mucosa layer (Table 1).

\section{Conclusion}

Our findings show that long-term NO inhibition significantly increases the sensitivity of DSM for the muscarinic 

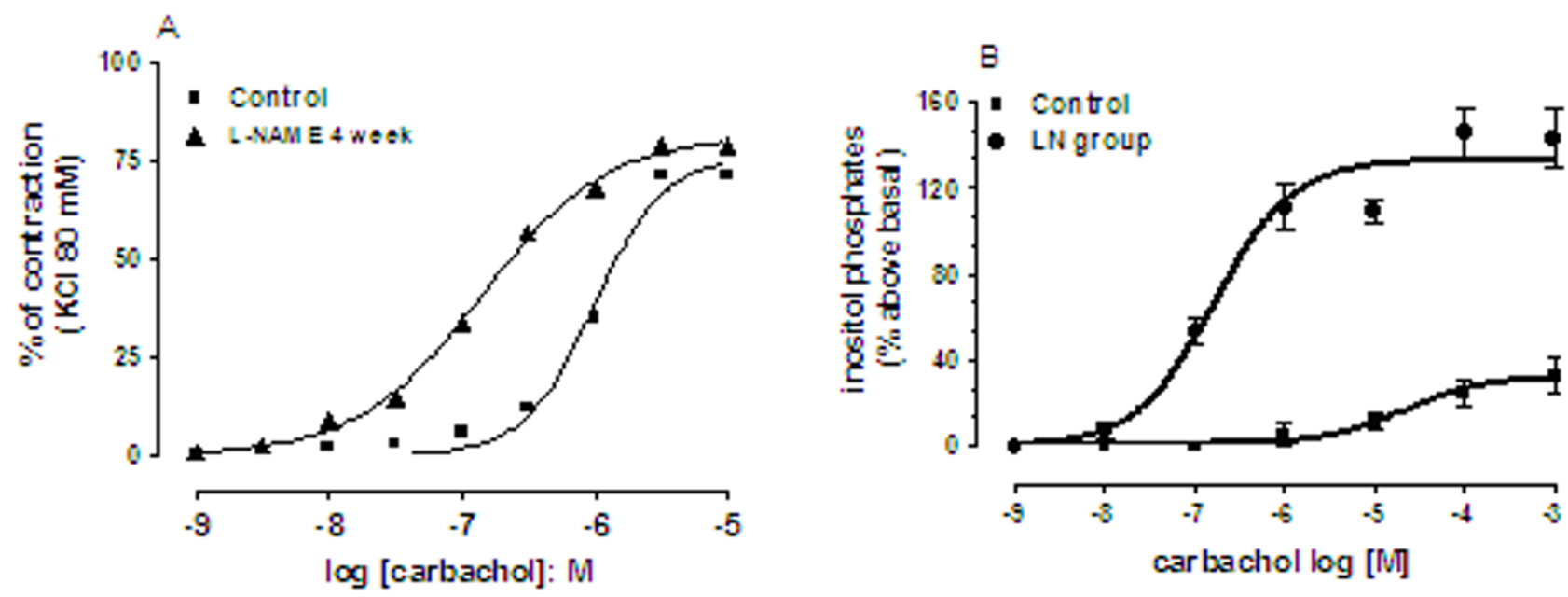

Figure I

(A) Concentration-response curve to carbachol in rat DSM from control and L-NAME-treated rats in 30 days ( $n=6-8, p<$ 0.05). (B) Effects of carbachol on total $\left[{ }^{3} \mathrm{H}\right]$-inositol phosphate accumulation in both control and L-NAME-treated group in 30 days $(n=3-6, P<0.05)$.

Table I: Morphometric analyses in isolated rat detrusor and trigone smooth muscle in control and L-NAME-treated groups in 30 days. $* \mathrm{p}<0.05$.

\begin{tabular}{|c|c|c|c|c|}
\hline & Detrusor $(\mu \mathrm{m})$ & & Trigone $(\mu \mathrm{m})$ & \\
\hline Layers & Control & L-NAME & Control & L-NAME \\
\hline Total & $1194 \pm 73.09$ & $1098 \pm 67.72$ & $705 \pm 19.56$ & $861 \pm 21.85^{*}$ \\
\hline Muscular & $610 \pm 37.18$ & $658 \pm 32.24$ & $526 \pm 24.29$ & $677 \pm 30.49^{*}$ \\
\hline Serosal & $547 \pm 66.36$ & $494 \pm 56.15$ & $176 \pm 12.47$ & $183 \pm 17.11$ \\
\hline
\end{tabular}

agonist carbachol via accumulation of $\mathrm{IP}_{3}$, suggesting that NO exerts a modulatory effect on the contractility mediated by muscarinic receptors.

\section{Acknowledgements}

Financial Support by FAPESP. 